\title{
OBSERVATIONS ON SOME PROBLEMS ENCOUNTERED IN THE ROUTINE PERFORMANCE OF THE DYE TEST FOR TOXOPLASMOSIS
}

\author{
BY \\ MORRIS GOLDMAN \\ From the Department of Health, Education, and Welfare, Communicable Disease Centre, Chamblee, Georgia, U.S.A.
}

(RECEIVED FOR PUBLICATION JULY 12, 1955)

The dye test for toxoplasmosis (Sabin and Feldman, 1948) depends upon the fact that toxoplasma organisms lose their affinity for methylene blue when they are exposed to specific antibody plus a heat-labile "accessory factor" at present available only from certain human sera. Accessory factor has been shown to differ from complement in several respects. Sera containing antibodies which produce the reaction are said to have "modifying activity" and toxoplasma cells which have been affected by the reaction are referred to as " modified organisms."

In trying to set up a routine for running the dye test for toxoplasmosis, it was found that results were often erratic and unpredictable. This has been the experience of other authors in widely different areas (Cathie and Dudgeon, 1953; Lelong and Desmonts, 1952). After taking particular precautions to assure that general laboratory procedures and glassware were not responsible for this situation, it was found that a percentage of routine tests was still erratic and that some had to be discarded because improper results were obtained with control sera.

An attempt was made to standardize the toxoplasma suspension which is added to the test sera by washing the organisms obtained from the peritoneal exudate of infected mice and replacing the liquid portion of the exudate with one of known composition.

Results obtained in preliminary experiments showed that washed organisms suspended in serumsaline were more sensitive to modification by serum than those suspended in the original exudate. This increased sensitivity was specific in so far as it was apparent only in the presence of active accessory factor. In addition, evidence was obtained that so-called negative human sera used for accessory factor often contained measurable amounts of a modifying substance. This paper presents a des- cription of this work and some observations concerning the dye test as a whole.

\section{Materials and Methods}

Dye test experiments were set up in which three types of toxoplasma suspensions were exposed to human sera in a block test type of arrangement. The percentage of modified organisms in the various tubes of these tests showed, on the one hand, whether the organisms were affected by the suspending media, and, on the other, whether the sera involved had accessory factor and/or modifying activity. The general method of performing the dye test was based on the procedure recommended by Sabin, Eichenwald, Feldman, and Jacobs (1952).

Preparation of Toxoplasma Suspensions.-A barbiturate-buffered saline solution (Kabat and Mayer, 1948) containing, in addition, $0.05 \% \mathrm{Na}$ thioglycollate and $10 \%$ inactivated human serum which was negative undiluted in the routine dye test was used as the washing and suspending medium for the organisms. The $p \mathrm{H}$ of the serum-saline medium was 7.3.

To study the effect of washing the organisms, three types of suspensions were used: suspension " A" consisted of toxoplasms in the original exudate. In suspension " B," the toxoplasms were first washed free of the liquid exudate and then were resuspended in this removed liquid. In suspension " $\mathrm{C}$," the toxoplasms were washed free of the liquid exudate and then were suspended in fresh serum-saline. The three suspensions were all prepared from one original batch of exudate and were all centrifuged and mechanically manipulated in the same manner. Manipulations followed this pattern: an initial centrifugation so that the supernatant liquid exudate could be removed for use in suspension " $\mathrm{B}$," suspension and centrifugation in 10 to 20 volumes of serum-saline, and finally resuspension in a small volume of liquid for use in the test. Centrifugation was at 1,500 r.p.m. for 5 to 10 minutes. Between 20 and 40 minutes elapsed from the time the exudate was withdrawn from mice and the time the suspensions were added to the sera and put into the water-bath. 
Sera Tested.-Eight sera, divided into two groups, were used in this study. In one group of six sera, all the donor individuals were adults who had been tested previously by routine dye tests performed either in this or other laboratories and found to be negative at least at the 1:16 level. Five of these sera had been used as sources of accessory factor in this or other laboratories, implying that they were negative undiluted or full strength. The second group consisted of two sera obtained from children of ages 4 and 9 . These had been submitted with 12 other samples from children to be tested for evidence of conditions other than toxoplasmosis.

In order to ascertain whether a given serum showed modifying activity, $0.08 \mathrm{ml}$. of the undiluted serum was incubated with $0.1 \mathrm{ml}$. of saline and $0.02 \mathrm{ml}$. of toxoplasma suspension ( $\mathrm{Ab}$ tube). Since a low count of modified cells in this tube could be due to lack of accessory factor activity as well as to lack of modifying activity, $0.08 \mathrm{ml}$. of the same undiluted serum was added to $0.1 \mathrm{ml}$. of a known positive serum and $0.02 \mathrm{ml}$. of toxoplasma suspension (positive control tube). The positive serum had previously been found to have a titre between 1:256 and 1: 1024. In the present series of tests, it was used in 1:100 dilution. In order to demonstrate that the modified count obtained in the first two tubes was due to a reaction involving accessory factor, a negative control was set up similar in all ways to the first tube except that the accessory factor activity of the serum was first destroyed by heating it for 30 minutes at $56^{\circ} \mathrm{C}$. (inactivated AF tube). An additional negative control was used which contained $0.18 \mathrm{ml}$. of saline plus $0.02 \mathrm{ml}$. of toxoplasma suspension (saline control tube). This last tube indicated the condition of the organisms when incubated in the absence of test serum following the mechanical manipulation of washing and centrifugation.

Statistical Evaluation of Organism Counts.-The recommended procedure for reading dye test results (Sabin et al., 1952) is to count 100-200 organisms at random in one preparation made from each tube, but before one could attempt to compare results obtained in parallel tests it was necessary to have some idea of the variation one could expect in counting stained and unstained organisms under the microscope.

A tube was chosen in which approximately half of the organisms were not stained. Ten separate counts were made from this tube, the staining reaction of 100 organisms in each count was tabulated, and the proportion of modified organisms was determined for the series. This procedure was repeated two more times using tubes which showed high and low counts of unstained organisms respectively. In each case the theoretical (binomial) standard deviation of the proportion was very close to the corresponding observed standard deviation. It was concluded, therefore, that the different counts obtained from any particular tube would vary from one another in a manner describable by the binomial distribution. In comparing counts obtained in different tubes, the observed count plus or minus 2 standard deviations was taken to represent the limits of the "true" count for each tube. When the difference between two tubes was

PERCENTAGE OF ORGANISMS MODIFIED IN THE DYE TEST USING THREE TYPES OF TOXOPLASMA SUSPENSION

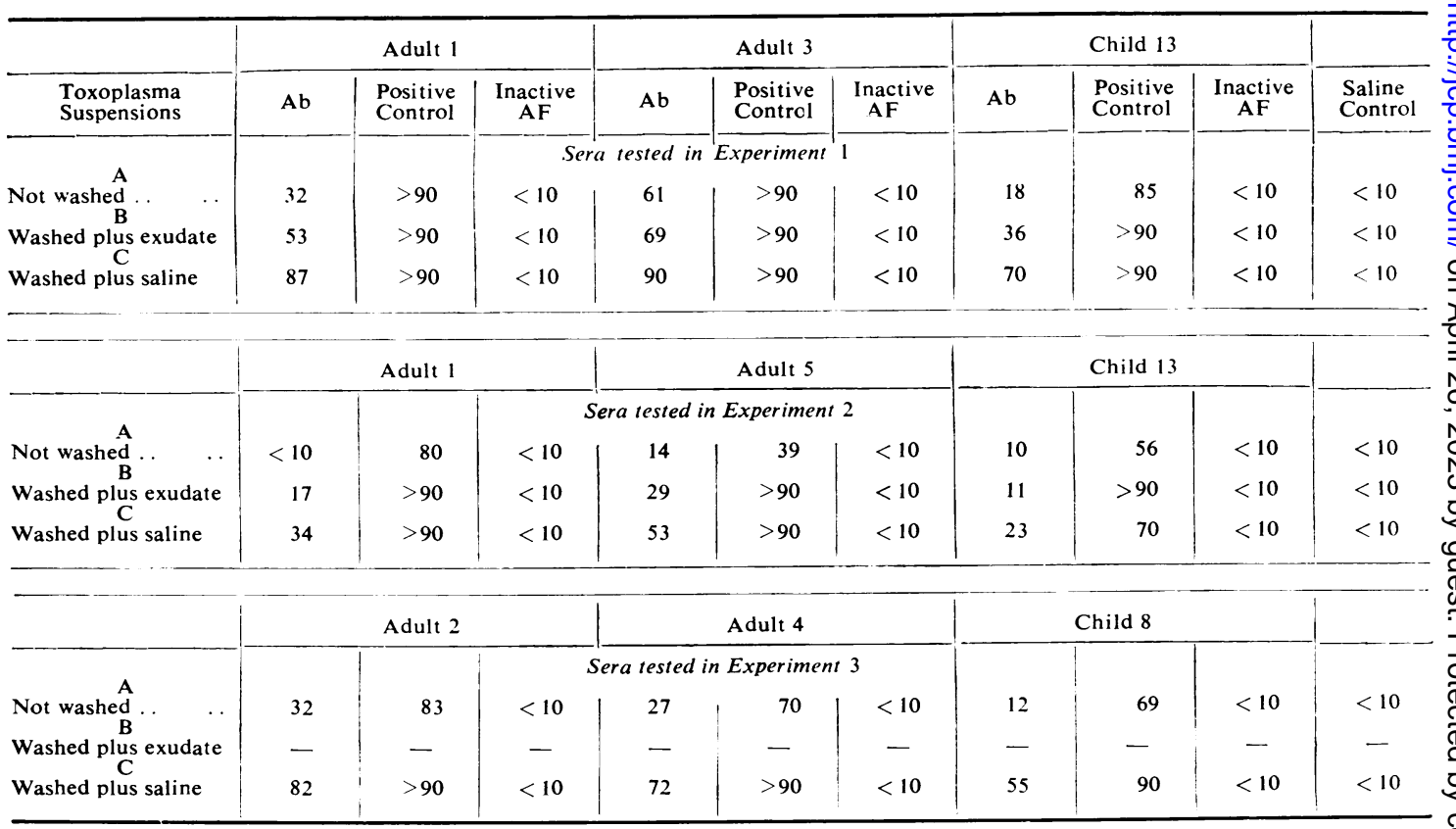


not clear-cut on this basis, the significance of the difference was tested by

$$
t=\frac{p_{1}-p_{2}}{\frac{\sqrt{p_{1}\left(1-p_{1}\right)}}{n_{1}}+\frac{p_{2}\left(1-p_{2}\right)}{n_{2}}}
$$

where $p_{1}$ and $p_{2}$ are the proportions stained of the $n_{1}$ and $\mathrm{n}_{2}$ examined in the two tubes. A value of $\mathrm{t}$ greater than 2 was considered to be statistically significant.

\section{Results}

Typical protocols showing results obtained when the three types of toxoplasma suspensions were used in the dye test are shown in Table I. Attention is directed primarily to the $A b$ tubes which show the percentage increase of modified toxoplasms when the liquid portion of the peritoneal exudate was replaced with serum-saline. That this effect was not due to non-specific damage because of handling is indicated by the low counts obtained in those tubes which did not contain accessory factor, i.e., in the saline and inactive AF tubes, regardless of type of suspension used. The pattern of results was the same whether the initial susceptibility of the organisms as indicated by suspension $\mathrm{A}$ was high (Experiment 1) or low (Experiment 2).

Significantly lower modified counts were found in two sera from children as compared with the sera obtained from adults except when the organisms were showing very low susceptibility to modification (Experiment 2, suspension A).

\section{Discussion}

When this work was begun, it was hoped that a means would be found to eliminate the day-to-day variation in results which was encountered. To date, however, it has only been possible to confirm, by different methods from those used by other workers, that the peritoneal exudate was a major cause of this variability. If the liquid portion, alone, of the exudate were responsible, one would expect that replacing this portion with a standard solution would make final results with different batches of exudate more uniform. In our hands this did not occur (Table I, suspension C). A plausible explanation for these results is that the toxoplasms themselves vary from time to time. Numerous experiments, of which the protocols reported here are typical, suggest strongly that, all other factors being equal, the number of toxoplasms modified in the dye test by any one serum depends largely upon the degree of susceptibility of the organisms in the exudate. Other workers have presented evidence that the liquid portion of the exudate may interfere with the test by containing either excess mouse antibody (Beverley and Beattie, 1952) or soluble toxoplasma antigen (Jacobs and Cook, 1954).

The percentage of modified toxoplasms encountered in the Ab tube, which is, in effect, a negative control, presents a problem. Beverley and Beattie (1952) consider that under the conditions of their tests this count is due to mouse antibody. In the experiments reported here, it was found that various " negative" sera containing similar accessory factor activity yielded significantly different results when mixed full strength with toxoplasma organisms. The pattern of the results suggested that it was serum antibody in very low concentration that was being measured in these tests. Harboe (1952) reported that only two out of $\mathbf{8 0}$ persons tested gave constantly less than $20 \%$ modified organisms when their serum was used as accessory factor with negative control sera. Cathie and Dudgeon (1953) reported, " On more than one occasion our so-called normal diluting serum has been shown to have a trace of antibody, sufficient to make the results equivocal." Surveys of large population groups (Hedqvist, 1953; Feldman, 1953, 1954; Beverley, Beattie, and Roseman, 1954) have shown that fewer children give positive tests for toxoplasmosis than adults. The fact that in the present experiments it was serum from adults that gave the higher counts and serum from children that gave the lower counts lends further support to the idea that human antibody activity was being observed.

At the present state of our knowledge the problem of standardizing the dye test for routine diagnostic work would seem to depend more upon standardizing the main ingredients, i.e., the organisms and accessory factor, rather than upon making specific techniques uniform in all laboratories. Use of toxoplasma grown in tissue cultures where the hostparasite relationship is simpler than that which exists in infected mice might result in a more reliable antigen than is now available. A good animal source of accessory factor would be very desirable. It would seem most promising to survey the serum of young animals of different species for such activity, since, by analogy with the situation in man, such individuals may be less likely to have developed antibodies.

The question of reproducibility of dye test results does not fall within the scope of this paper, since no systematic attempts were made to study that problem. However, replicate tests on the same sera performed in connexion with this and other problems sometimes showed disturbing anomalies. Published reports on this point by other workers are meagre. In one series of seven sera examined by two laboratories (Beverley and Beattie, 1952), 
titres agreed closely in five cases; in two cases titres differed from two- to 10-fold. (Due to variation in reporting results, the precise differences between the sera could not be determined.) As another step in standardizing dye test results, it would be desirable to have a large group of sera tested independently by several laboratories under controlled conditions in order to see how much agreement is possible.

It would help, in interpreting dye test titres, if authors would indicate the types of controls used and the results obtained in such controls. It would be especially important to know what use is made of the modified count, if any, in the negative control tube. Beverley and Beattie (1952), for example, take as the titre for a serum the highest dilution which will modify $50 \%$ of the toxoplasms beyond the number which are affected in the negative control if the latter is less than 20 . In the procedure recommended by Sabin et al. (1952) the count in this control is not taken into consideration in determining serum titre. Feldman (1954) considers that a modified count greater than $10 \%$ renders the test unsatisfactory. Obviously, a count of, for example, $15 \%$ modified organisms in the negative control might not necessarily invalidate the test, but it might change a serum titre, depending upon how it was used.

\section{Summary}

When the liquid portion of mouse peritoneal exudate containing toxoplasms was replaced with a

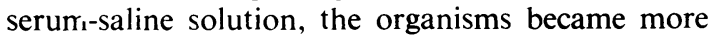
sensitive to the modifying action of "negative" sera containing accessory factor. When the accessory factor was destroyed by heating, the organisms were not modified by the same sera.

Modifying activity of "negative" human sera examined in this study appeared to be due to antibody in low concentrations. Serum obtained from two out of 14 children showed significantly less modifying activity than the six " negative" adult sera studied.

In this laboratory, it appears that major variations in dye test results are due to inherent variability of the peritoneal exudate which is obtained from infected mice.

Suggestions are made concerning some ways by which the dye test might be better standardized for routine use in diagnostic laboratories.

This work was performed in the Toxoplasma Diagrostic Laboratory of the Communicable Disease Centre, Dr. M. M. Brooke in charge. Grateful acknowledgment is made to Dr. Brooke for permission to use the laboratory and to Mr. A. J. Sulzer, who kindly provided the materials necessary to perform the experiments.

\section{RFFERENCES}

Beverley, J. K. A., and Beatiie, C. P. (1952). Journal of Clinical Pathology, 5, 350 .

Catho Roseman, C. (1954). J. Hyg., Camb., 52, 37.

Cathie, I. A. B., and Dudgeon, J. A. (1953). Gt Ormond Str. J., 3 (No. 5), 13.

Feldman. H. A. (1953). Amer. J. trop. Med. Hyg., 2, 420.

- (1954). Bibl. ophthal. (Basel), 3, 1

Harboe, A. (1952). Acta path. microbiol. scand., Suppl. 93, p. 325

Hedquist, T. (1953). J. Hyg., Camb., 51, 478.

Jacobs, L., and Cook, M. K. (1954). Amer. J. trop. Med. Hyg., 3 , 860 .

Kabat, F. A., and Mayer, M. M. (1948). Experimental Immunochemistry. Thomas, Springfield, Illinois.

L.elong, M., and Desmonts, G. (1952). C.R. Soc. Biol. (Paris). 146 207.

Sabin, A. B., and Feldman, H. A. (1948). Science, 108, 660

Eichenwald, H., Feldman, H. A., and Jacobs, L. (1952), J Amer. med. Ass., 150, 1063 . 